\title{
* Fermilab
}

\section{SBND-PRISM: Sampling Multiple Off-Axis Fluxes with the Same Detector}

Marco Del Tutto, for the SBND collaboration In collaboration with Pedro Machado, Kevin Kelly, and Roni Harnik

APS April Meeting 2021 


\section{The Short Baseline Near Detector (SBND)}

SBND is the near detector in the Short Baseline Neutrino (SBN) program at Fermilab

Three Liquid Argon Time Projection Chamber (LArTPC) detectors located along the Booster Neutrino Beamline (BNB) at Fermilab

Goals of the SBN program:

Search for eV mass-scale sterile neutrinos oscillations

Study of neutrino-argon interactions at the GeV energy scale

Search for new/rare physics processes in the neutrino sector and beyond

\section{Target}

SBND

MicroBooNE

ICARUS

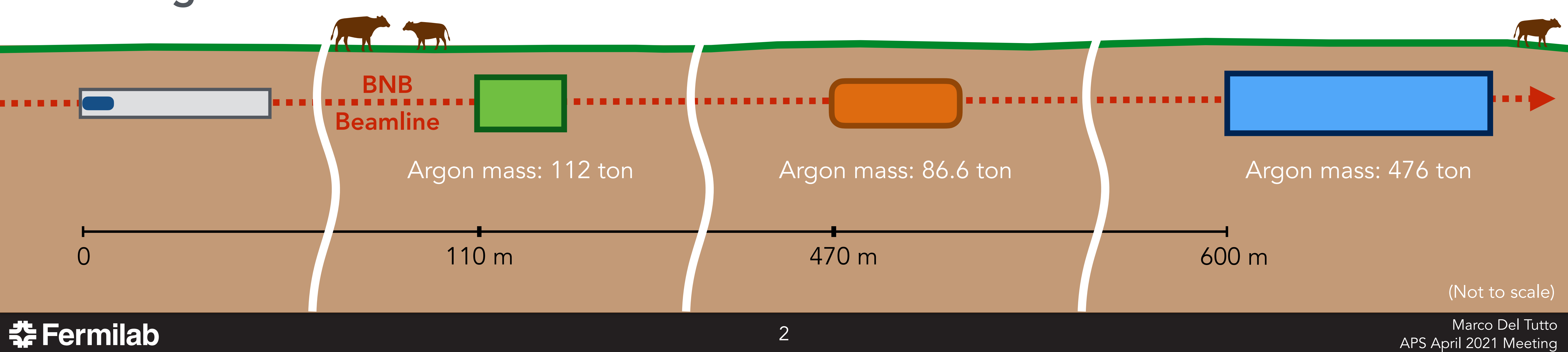




\section{A Slightly Off-Axis Detector}

\section{The SBND detector}

Cosmic Ray Tagger

CRT

SBND will be surrounded by scintillator strips to tag cosmic rays

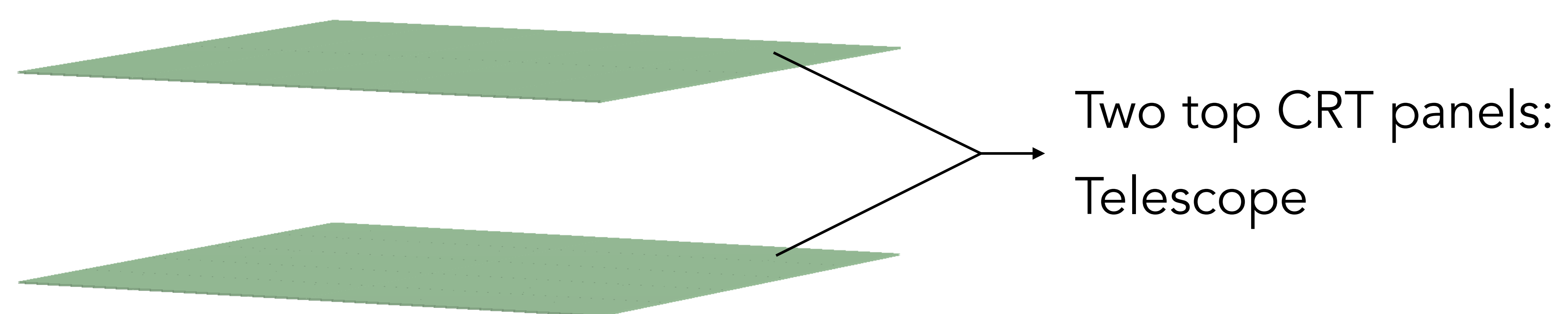

\section{g}

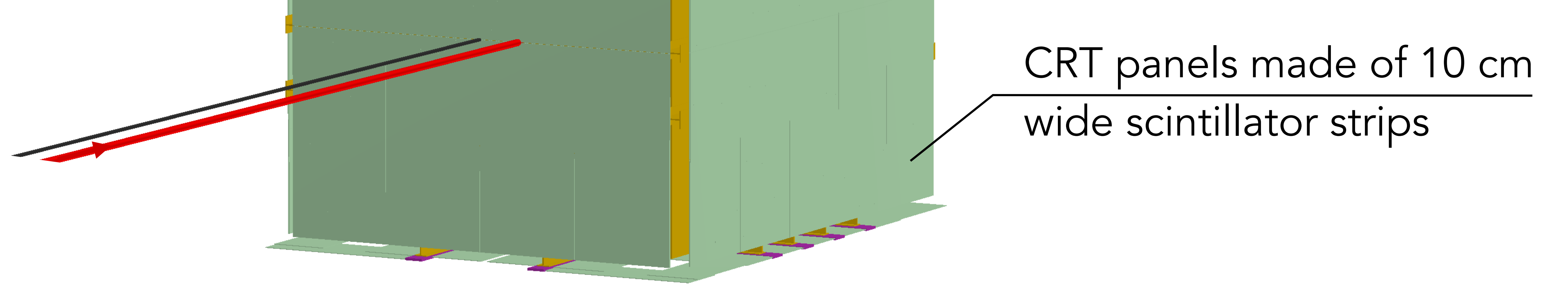




\section{A Slightly Off-Axis Detector}

\section{The SBND detector}

- Made of two liquid argon time projection chambers.

- 112 ton of liquid argon.

- Dimensions: $4 \mathrm{~m} \times 4 \mathrm{~m} \times 5 \mathrm{~m}$.

- $110 \mathrm{~m}$ from the target position.

- SBND is currently being installed. 


\section{A Slightly Off-Axis Detector}

\section{The SBND detector}

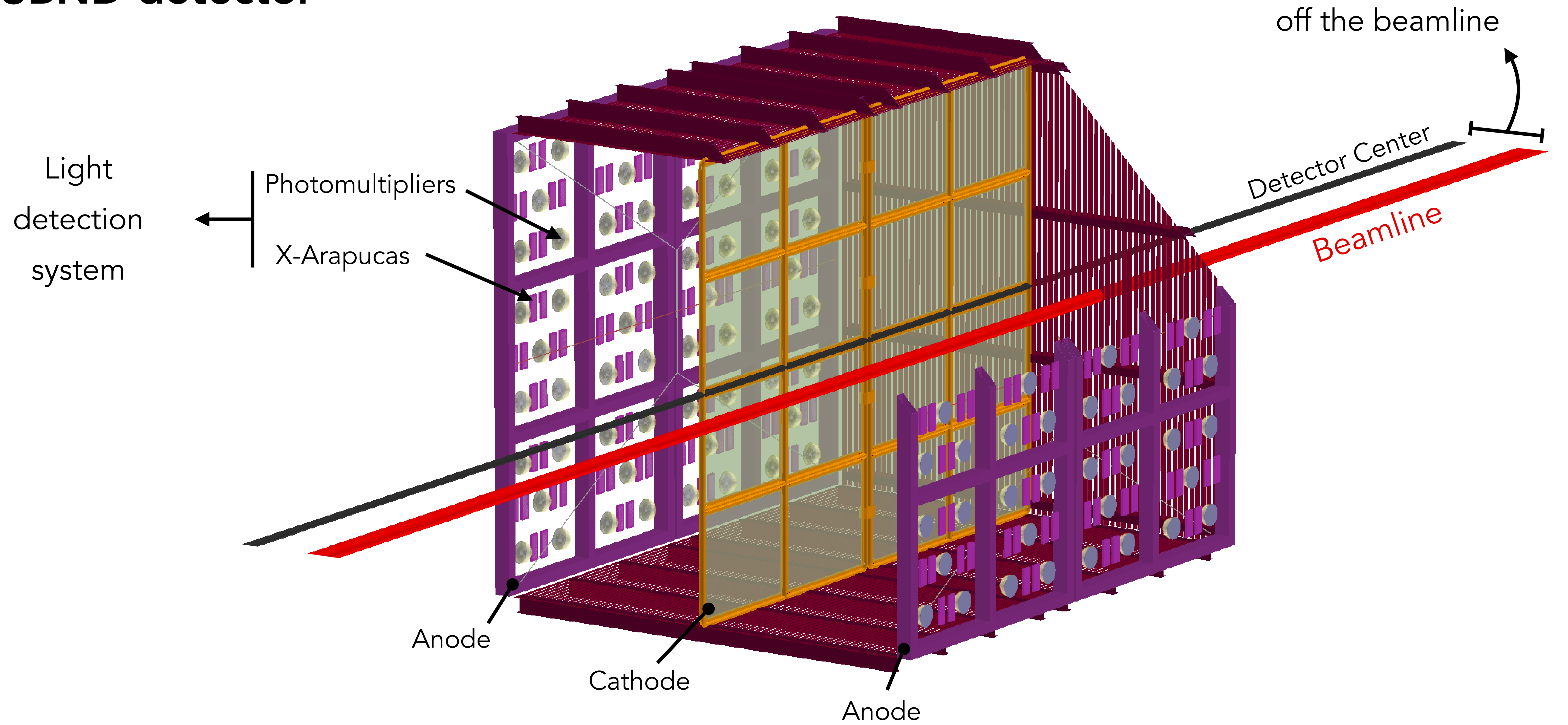

The detector is $\sim 74 \mathrm{~cm}$ off the beamline 


\section{A Slightly Off-Axis Detector}

SBND sees neutrinos from several off-axis angles (Off-axis angle is calculated w.r.t. target position)

The detector can be divided in several off-axis slices:

$\mathrm{OAA} \in\left[0.0^{\circ}, 0.2^{\circ}\right)$

$O A A \in\left[0.2^{\circ}, 0.4^{\circ}\right)$

$\mathrm{OAA} \in\left[0.4^{\circ}, 0.6^{\circ}\right)$

$\mathrm{OAA} \in\left[0.6^{\circ}, 0.8^{\circ}\right)$

$\mathrm{OAA} \in\left[0.8^{\circ}, 1.0^{\circ}\right)$

$\mathrm{OAA} \in\left[1.0^{\circ}, 1.2^{\circ}\right)$

$\mathrm{OAA} \in\left[1.2^{\circ}, 1.4^{\circ}\right)$

$\mathrm{OAA} \in\left[1.4^{\circ}, 1.6^{\circ}\right)$

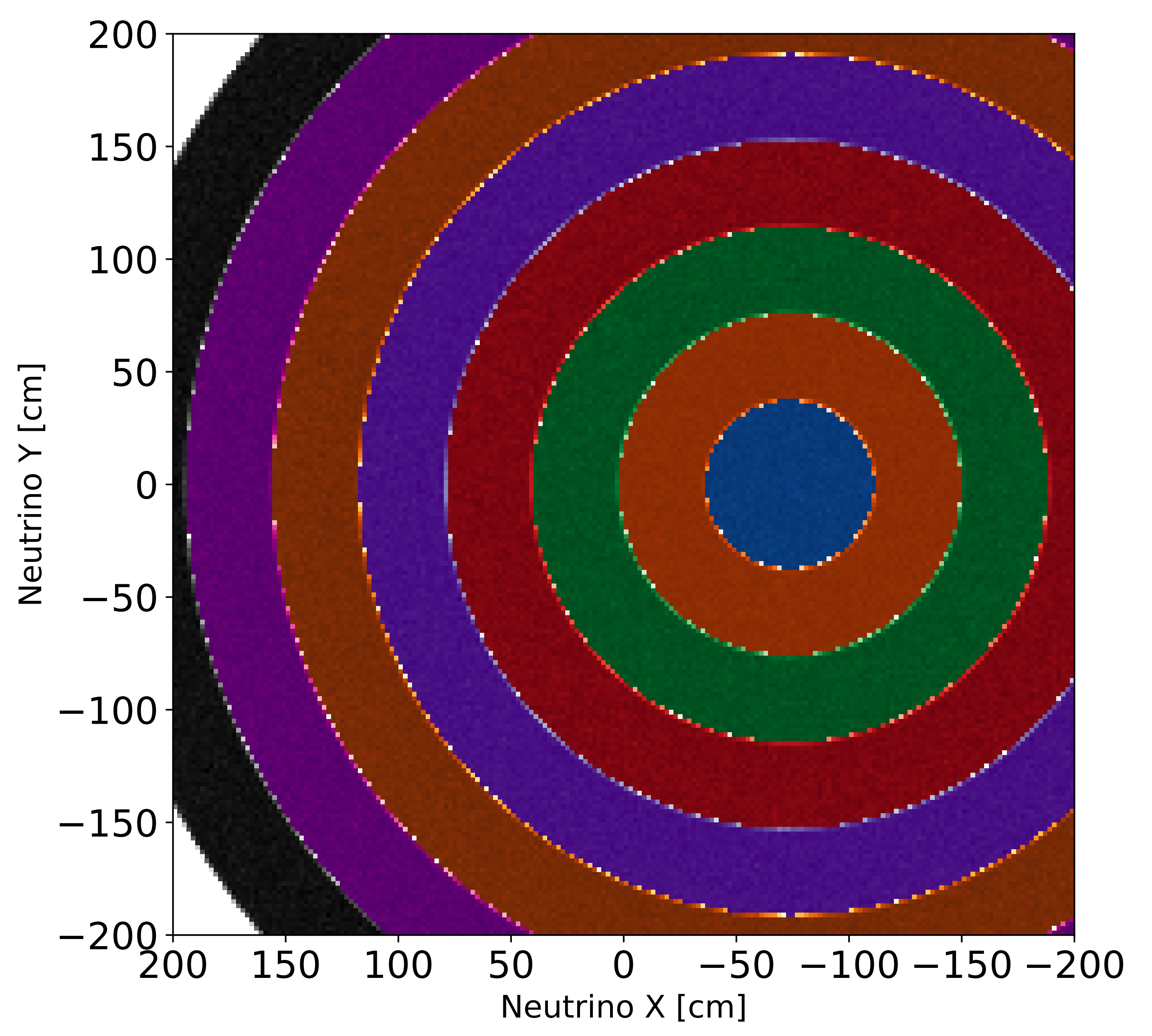




\section{SBND-PRISM}

Precision Reaction Independent $\mathbf{S}$ pectrum Measurement $\left.{ }^{*}\right)$

The $v_{\mu}$ energy distribution is affected by the off-axis position

Mean neutrino energy

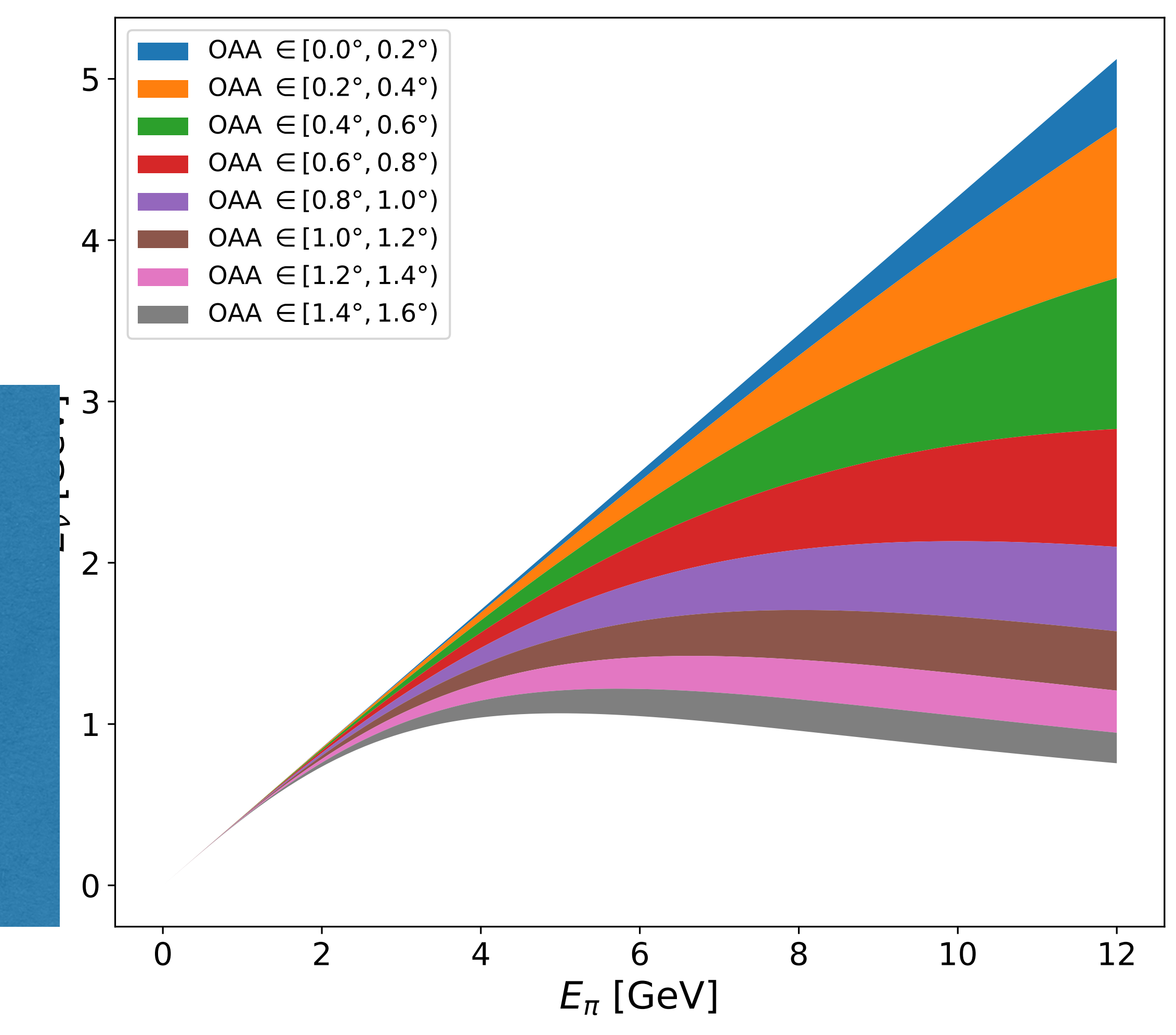

Muon neutrino flux in each

of the OAA regions

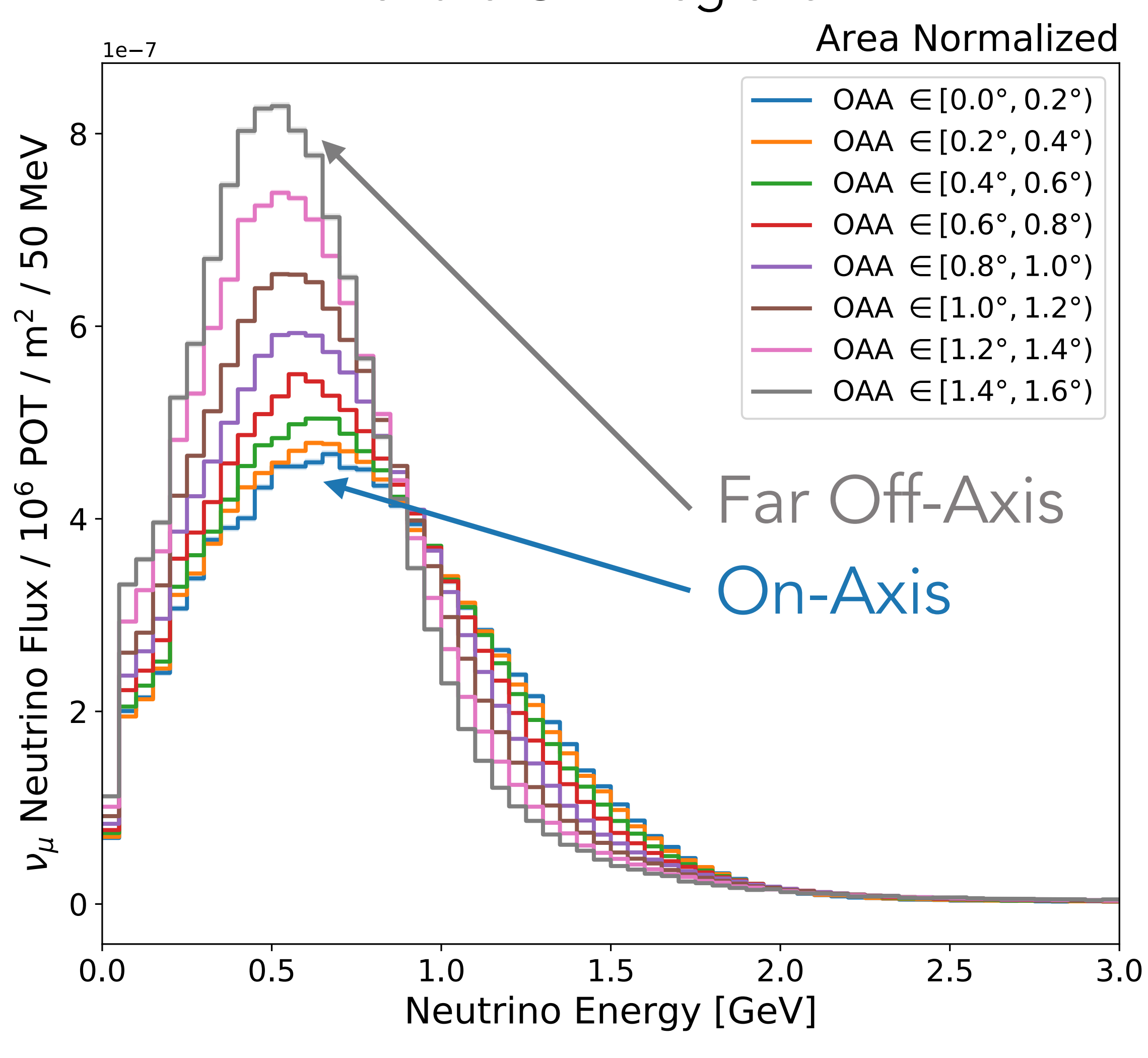

Neutrino events are

divided based on the off-axis angle (OAA) region they fall in:

$\mathrm{OAA} \in\left[0.0^{\circ}, 0.2^{\circ}\right)$

$\mathrm{OAA} \in\left[0.2^{\circ}, 0.4^{\circ}\right)$

$\mathrm{OAA} \in\left[0.4^{\circ}, 0.6^{\circ}\right)$

$\mathrm{OAA} \in\left[0.6^{\circ}, 0.8^{\circ}\right)$

$\mathrm{OAA} \in\left[0.8^{\circ}, 1.0^{\circ}\right)$

$\mathrm{OAA} \in\left[1.0^{\circ}, 1.2^{\circ}\right)$

$\mathrm{OAA} \in\left[1.2^{\circ}, 1.4^{\circ}\right)$

$\mathrm{OAA} \in\left[1.4^{\circ}, 1.6^{\circ}\right)$ 


\section{SBND-PRISM}

Precision Reaction Independent $\mathbf{S}$ pectrum Measurement ${ }^{*}$ )

The $v_{\mu}$ energy distribution is affected by the off-axis position

Mean neutrino energy

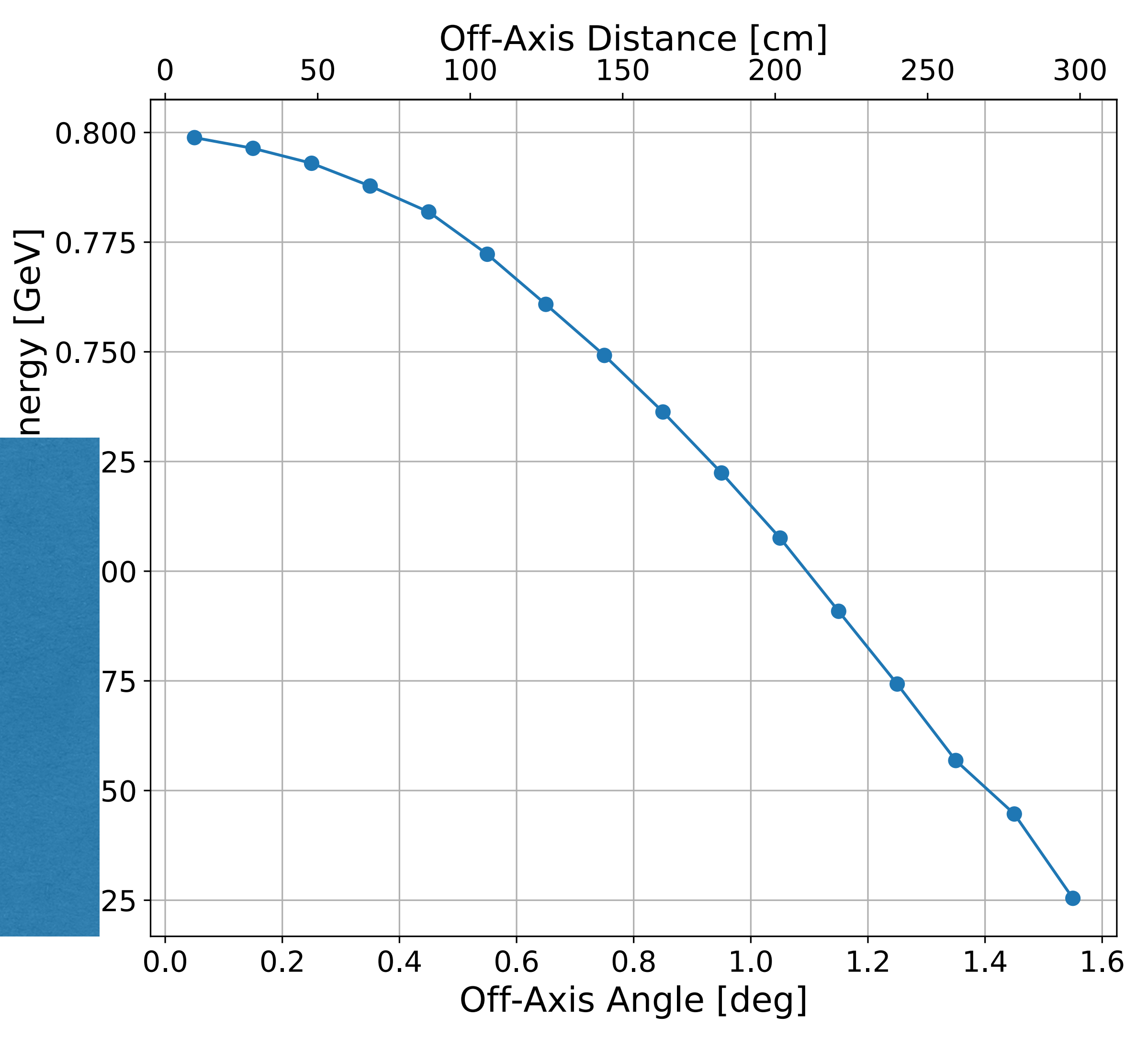

Muon neutrino flux in each

of the OAA regions

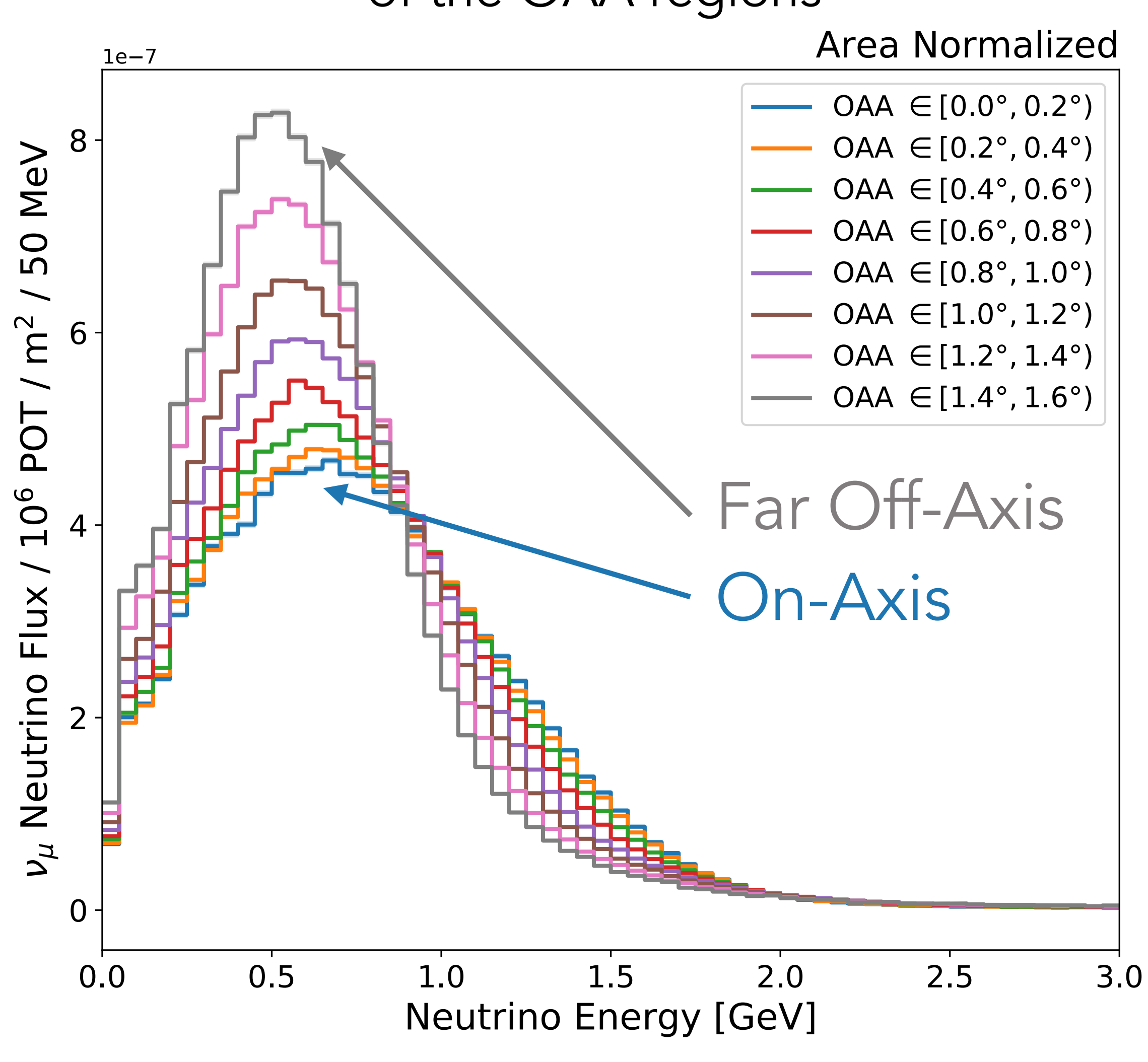

Neutrino events are

divided based on the off-axis angle (OAA) region they fall in:

$\mathrm{OAA} \in\left[0.0^{\circ}, 0.2^{\circ}\right)$

$\mathrm{OAA} \in\left[0.2^{\circ}, 0.4^{\circ}\right)$

$\mathrm{OAA} \in\left[0.4^{\circ}, 0.6^{\circ}\right)$

$\mathrm{OAA} \in\left[0.6^{\circ}, 0.8^{\circ}\right)$

$\mathrm{OAA} \in\left[0.8^{\circ}, 1.0^{\circ}\right)$

$\mathrm{OAA} \in\left[1.0^{\circ}, 1.2^{\circ}\right)$

$\mathrm{OAA} \in\left[1.2^{\circ}, 1.4^{\circ}\right)$

$\mathrm{OAA} \in\left[1.4^{\circ}, 1.6^{\circ}\right)$ 


\section{SBND-PRISM $-v_{\mu} / v_{e}$ Differences $1 / 2$}

Muon neutrino energy spectrum changes with the off-axis angle, while the electron neutrino one stays almost the same

\section{Muon-neutrino CC Events}

higher off-axis angle $\rightarrow$ lower mean energy

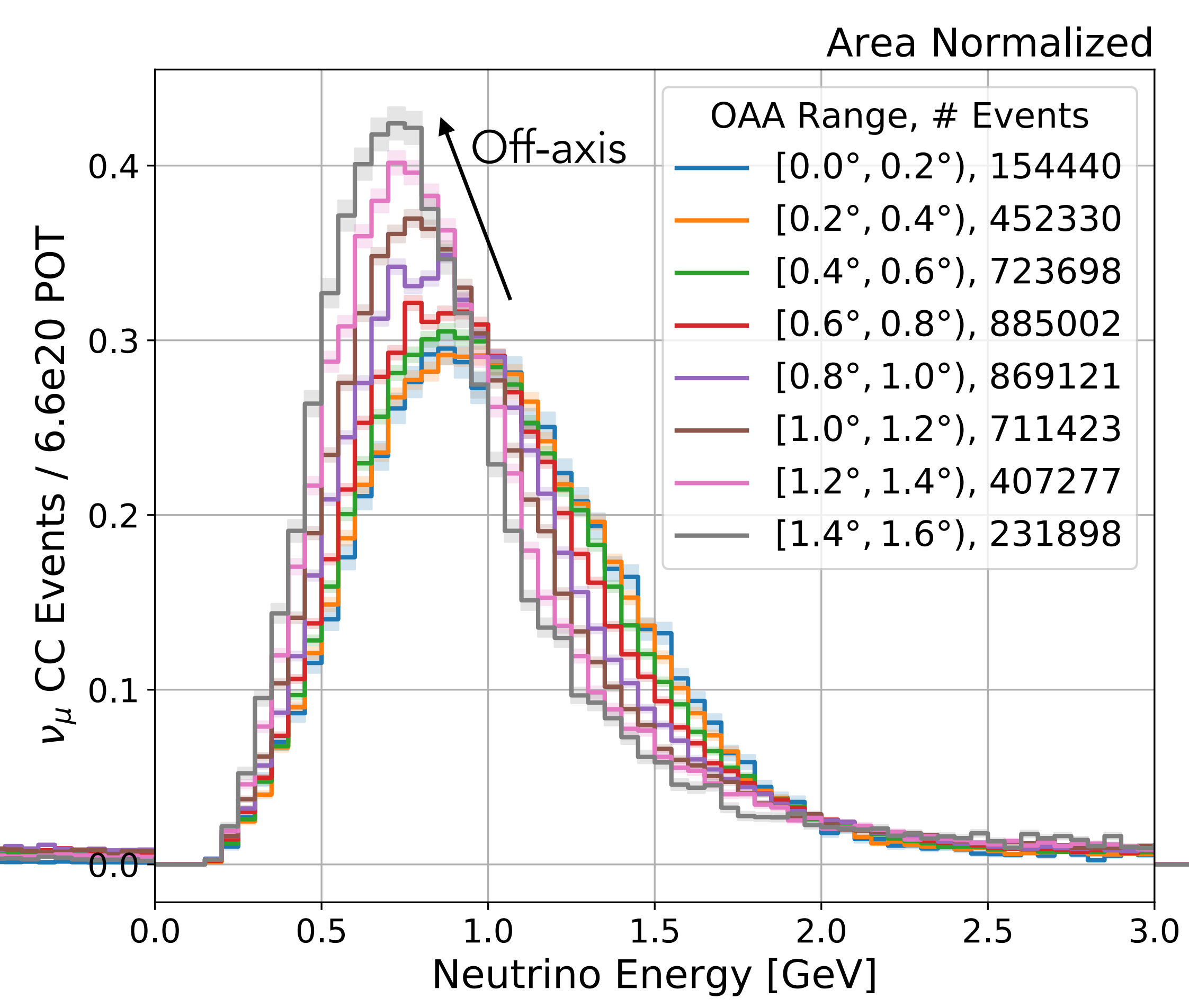

\section{Electron-neutrino CC Events}

higher off-axis angle $\rightarrow \sim$ same mean energy

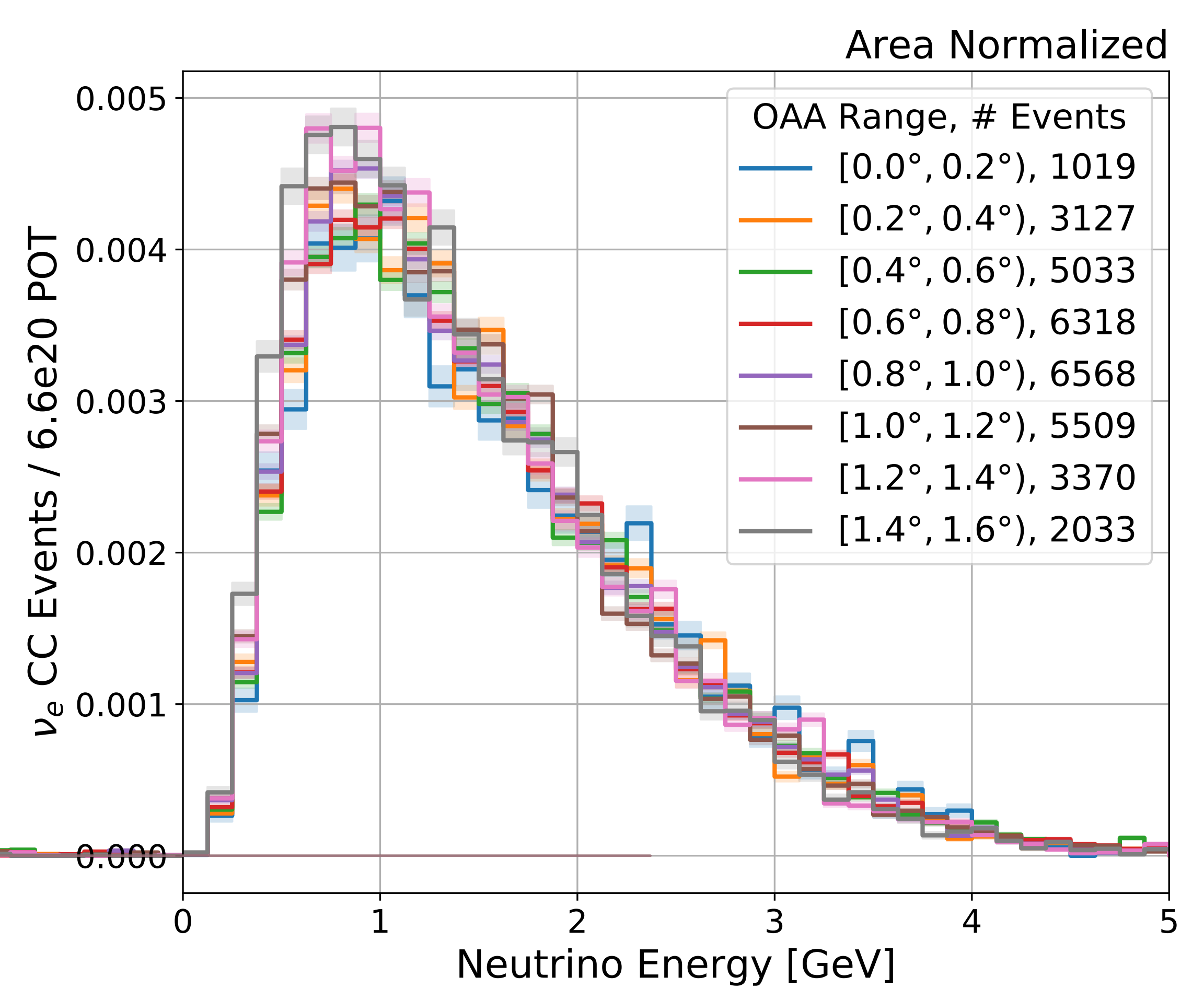

High event statistics in all off-axis regions 


\section{SBND-PRISM $-v_{\mu} / v_{e}$ Differences $2 / 2$}

Moving away from the beam-line axis, the number of $\nu_{\mu}$ and $\nu_{e}$ interactions varies differently. While the number of $\nu_{e}$ events stays almost constant, the number of $\nu_{\mu}$ events decreases.

\section{Muon-neutrinos CC Events}

peak coincident with the on-axis position

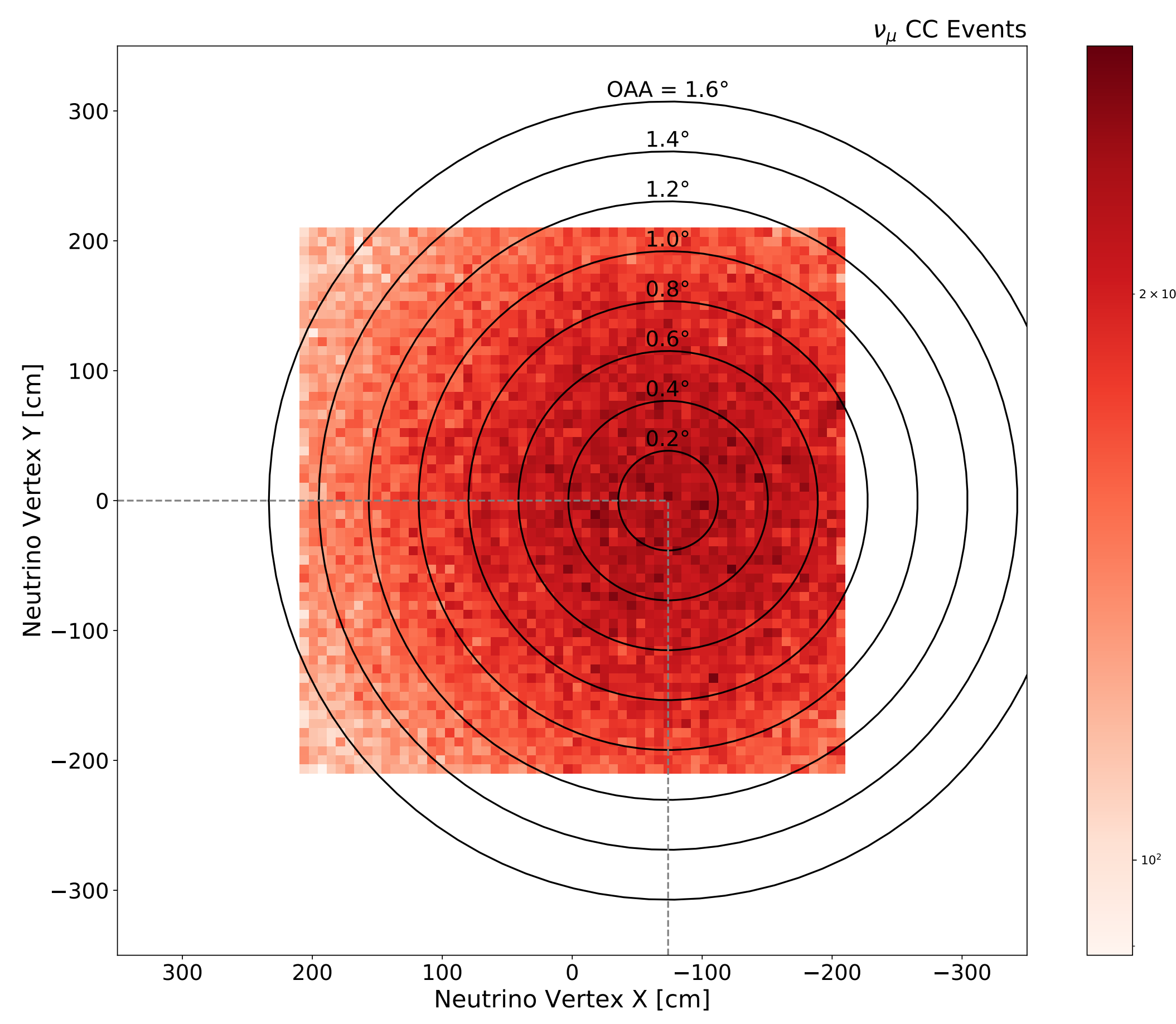

\section{Electron-neutrinos CC Events}

distribution is almost constant (angular distribution of $v_{e}$ is wider due to three-body decay)

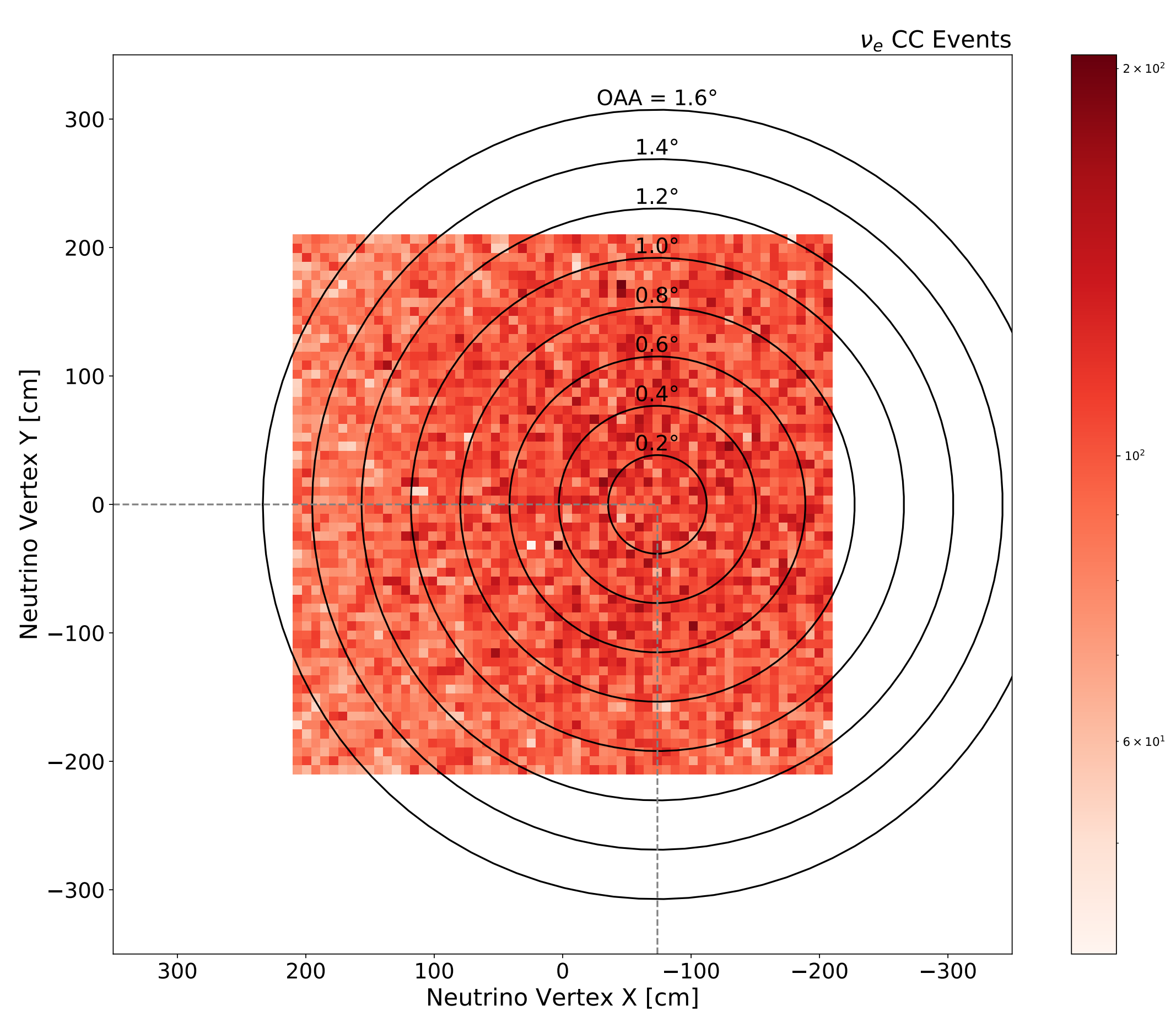




\section{Cosmic Ray Tagger Data}

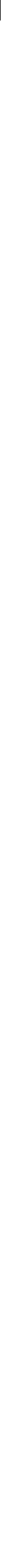




\section{SBND-PRISM - Applications}

\section{Benefits of SBND-PRISM:}

- Interaction Model Constraint

- Neutrino Oscillations

- Dark Matter Searches 


\section{SBND-PRISM - Interaction Model Constraint}

The PRISM feature of SBND opens up new analyses:

- Can make neutrino cross section measurements over a peak/mean energy that spans over 200 MeV energy difference (test of models/generators).

- Study the relationship between neutrino energy and lepton (and hadron) kinematics, done by measuring differential cross-section in lepton (and hadron) kinematics at different beam angles (= different neutrino energies).

- $\nu_{\mu}$ to $\nu_{e}$ cross section ratio: going off-axis, the increase in $\nu_{e}$ to $\nu_{\mu}$ flux ratio combined with a choice of kinematics where $\nu_{e}$ to $\nu_{\mu}$ differences are prominent should allow us to measure the $\nu_{e} / \nu_{\mu}$ cross section (can study lepton mass effects).
v-Ar CC Events

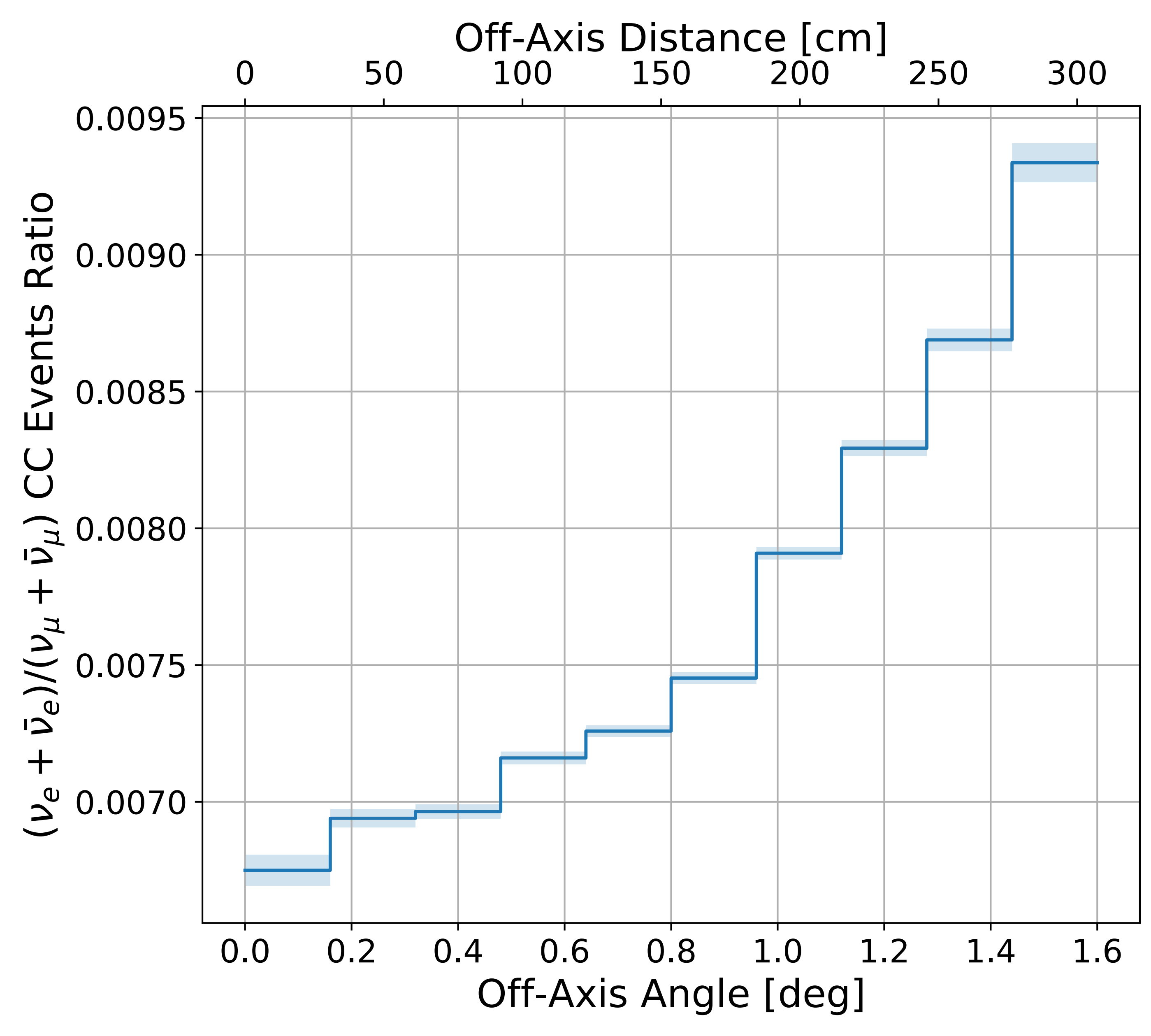




\section{SBND-PRISM - Sterile Neutrino Oscillations}

The PRISM feature of SBND can potentially improve the SBN sensitivities to sterile neutrino oscillations. Two possibilities to use the PRISM feature:

1. Instead of treating SBND as a single detector, we can treat it as multiple detectors at different off-axis positions and include those in the SBN oscillation fit. Since the the energy spectra are different the neutrino interaction model will be over constrained.

2. Can linearly combine the measurements the different off-axis positions to reproduce a given choice of incident neutrino flux. Can match the ICARUS (far detector) oscillated spectrum in SBND (near detector.)

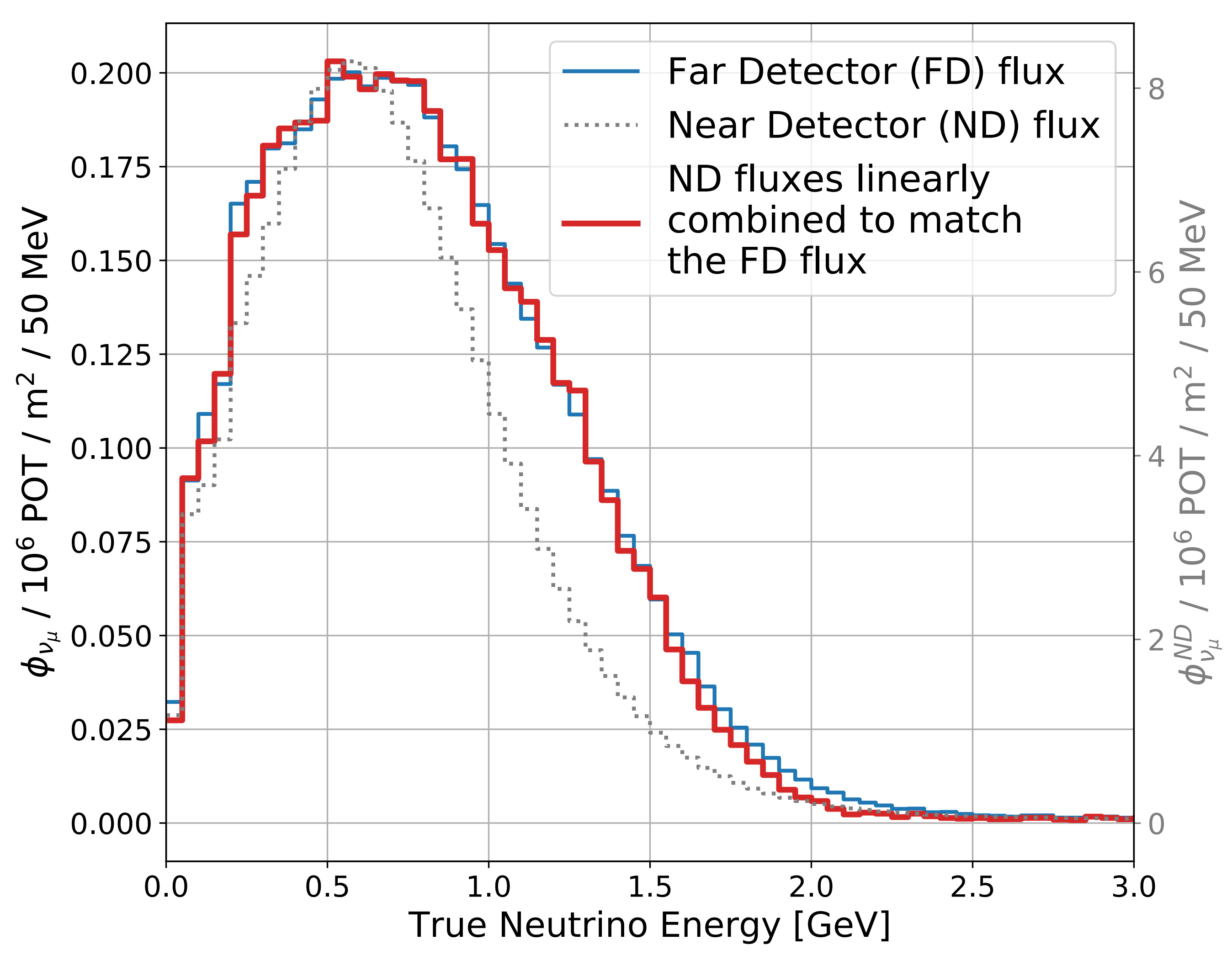




\section{SBND-PRISM - SBND-Only Sterile Neutrino Oscillations}

Can we use SBND-PRISM for SBND-only sterile neutrino searches?

SBND-PRISM potentially allows probing higher values of $\Delta \mathrm{m}^{2}$ for sterile neutrino oscillation searches.

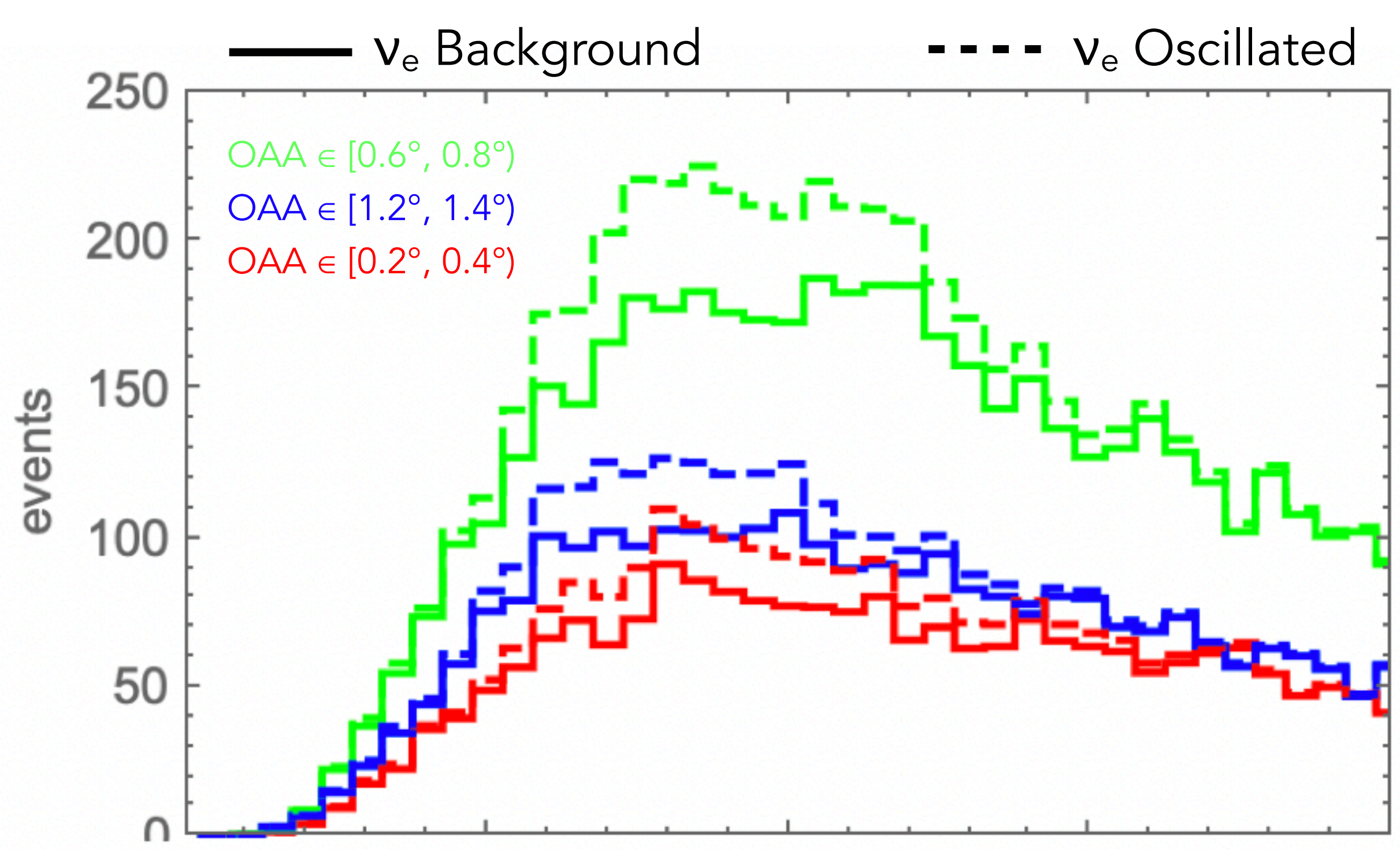

Testing sensitivity with:

- $\Delta \mathrm{m}^{2}=10 \mathrm{eV}{ }^{2}, \sin ^{2} 2 \theta_{\mu \mathrm{e}}=0.001$

- $v_{e}$ appearance mode

- very conservative systematics: free norm. $+30 \%$ bin-by-bin sys. on bkg

$$
\chi^{2}=\sum_{i, j}^{\text {pos., bins } \frac{\left(N_{i j}+\alpha T_{i j}\right)^{2}}{N_{i j}+\sigma_{b i n}^{2} N_{i j}^{2}}} \quad \begin{aligned}
& \text { w/ PRISM X2: } 13 \\
& \text { w/o PRISM X2: } 2
\end{aligned}
$$

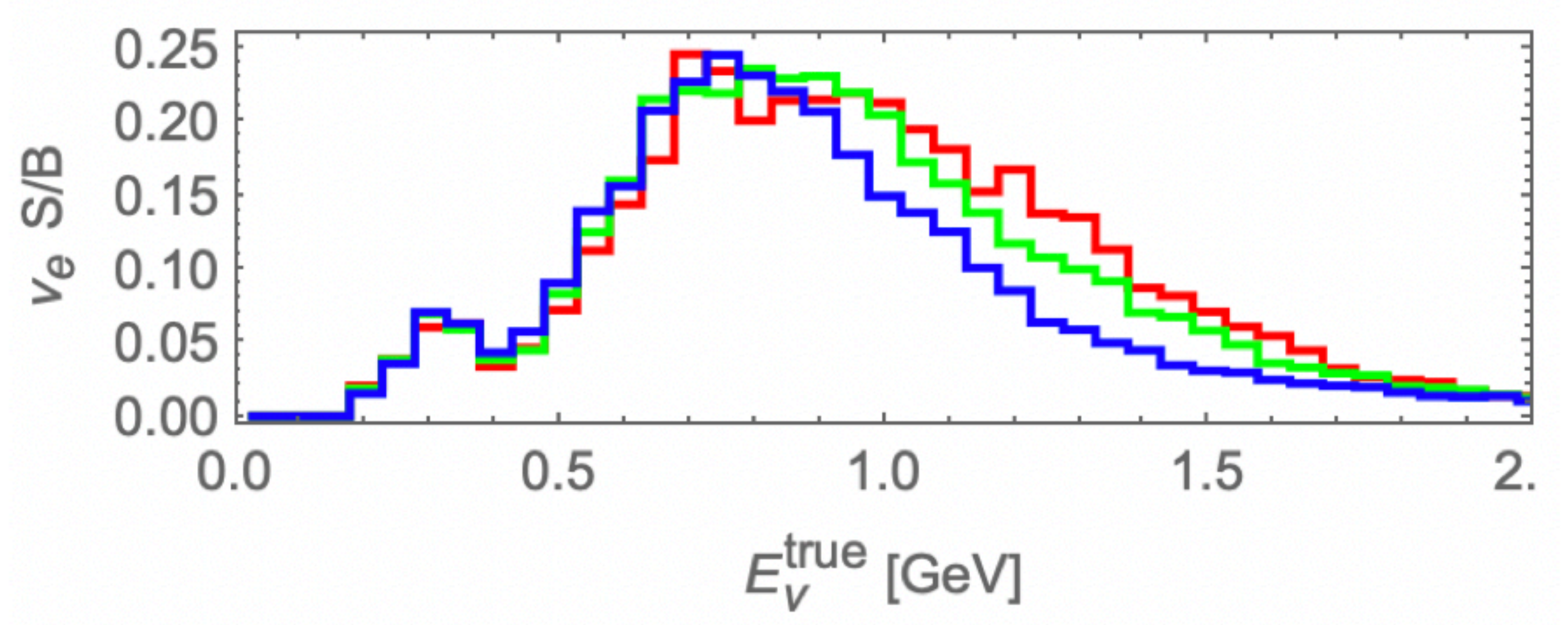

- Mismatch between $v_{\mu}$ flux and $v_{e}$ contamination on different off-axis positions may be an opportunity to do physics.

- Proper estimation of systematics is needed before final conclusions can be made, but results look promising with current (conservative) systematic guess. 


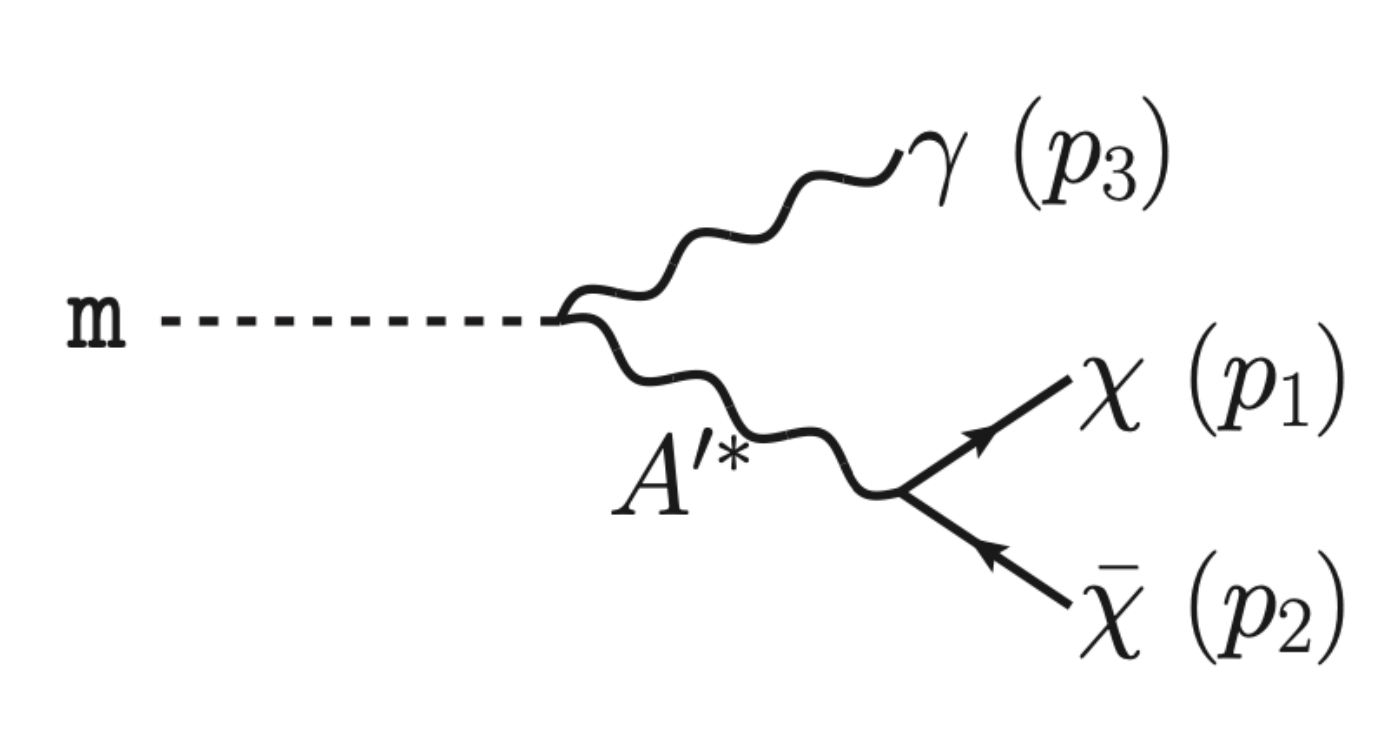

One example: light dark matter (sub-GeV) that is coupled to the Standard Model via a dark photon. The dark photons can be produced by neutral meson decays (pions, etas) in the target, and then decay to the dark matter. The dark matter can then travel to SBND and, through the dark photon, scatter off electrons in the detector.

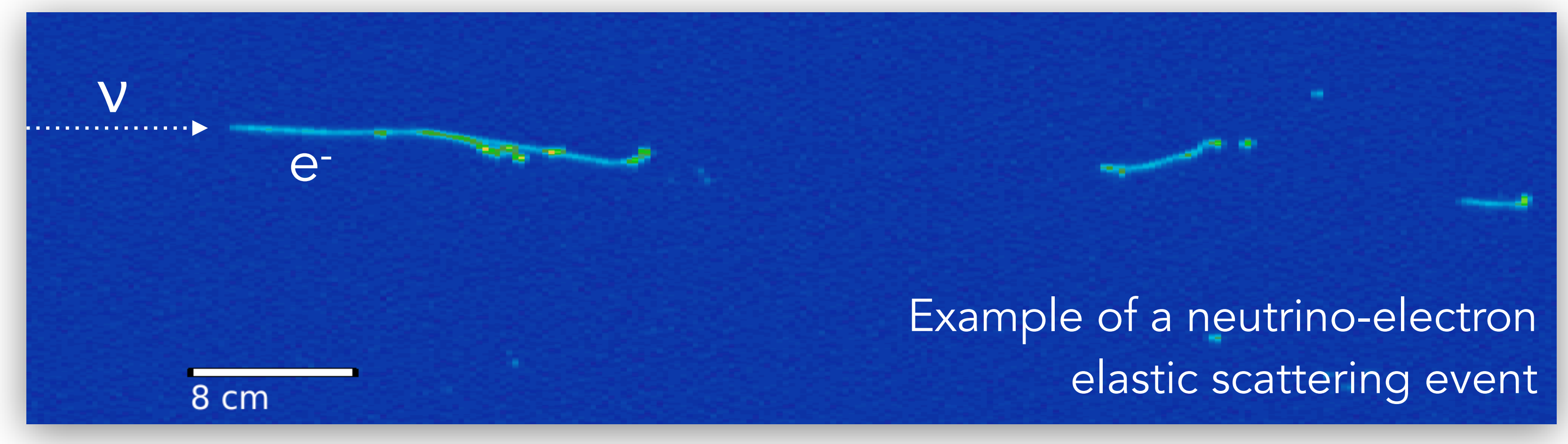

- Background: neutrino-electron elastic scattering. Neutrinos come from two-body decays of charged (focused) mesons.

- Signal: elastic scattering electron events. Dark matter comes from three-body decays of neutral (unfocused) mesons.

- Neutrino flux drops off more sharply as a function of radius! 


\section{Conclusions}

- The closeness of SBND to the neutrino source, combined with the abundance of statistics allows us to use this "free" PRISM feature.

- Contrary to DUNE-PRISM, SBND can take data on all the off-axis regions simultaneously, no need to move the detector.

- SBND-PRISM could constrain the relationship between true and reconstructed energy and potentially help disentangling the effects due to mismodeling of the neutrino flux, neutrino interaction crosssections, and detector response

- SBND-PRISM opens up new possibilities: can potentially constrain interaction modeling, improve oscillation fits, allows for an SBND-only oscillation analysis, and other BSM searches. 


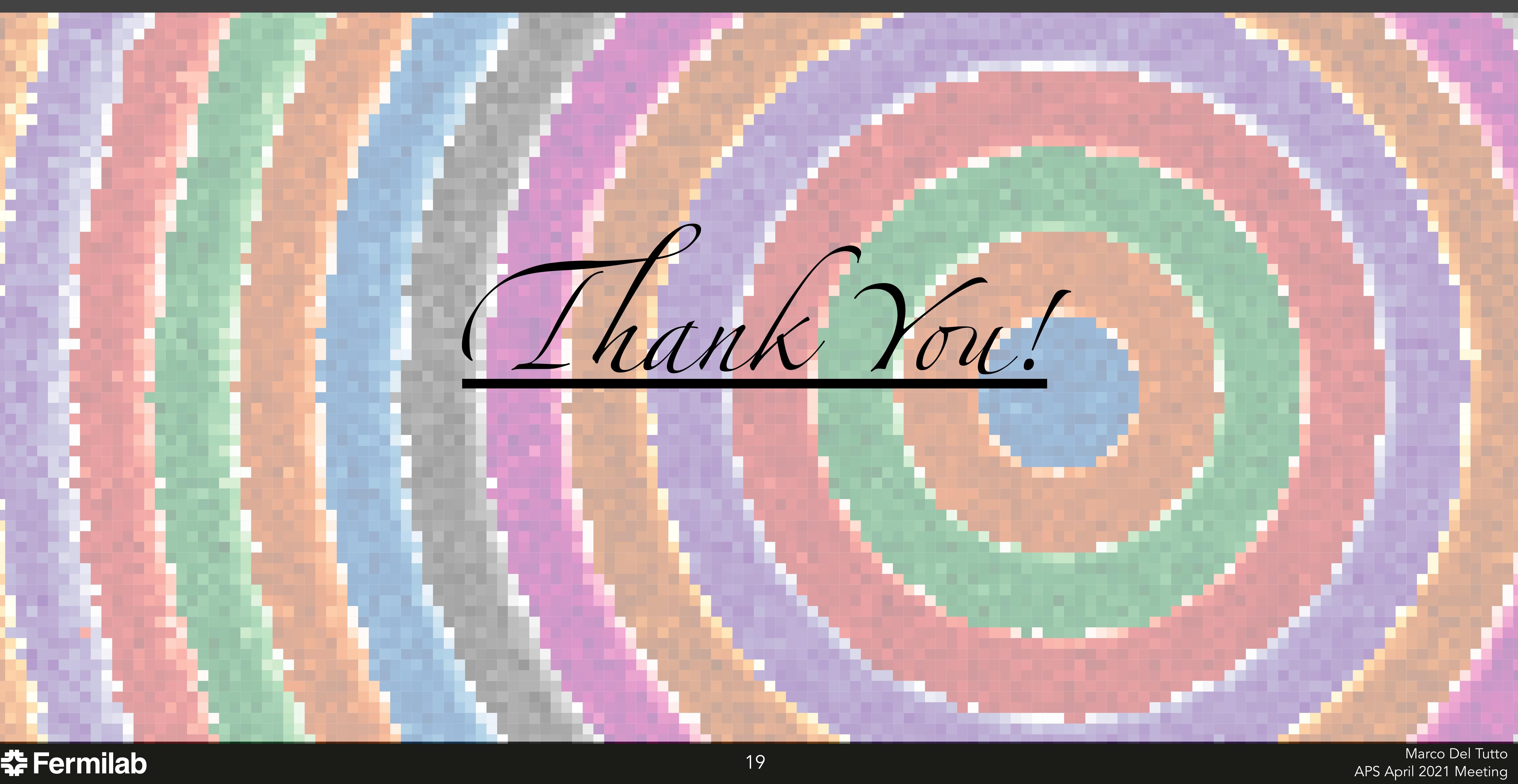

